The Astrophysical Journal, 547:1109-1115, 2001 February 1

(C) 2001. The American Astronomical Society. All rights reserved. Printed in U.S.A.

\title{
HUGE CORONAL STRUCTURE AND HEATING CONSTRAINTS DETERMINED FROM SERTS OBSERVATIONS
}

\author{
D. A. FALCONER ${ }^{1}$ \\ University of Alabama, Huntsville \\ AND \\ J. M. DAVILA \\ Goddard Space Flight Center/NASA, Laboratory for Astronomy and Solar Physics, Code 682, Greenbelt, MD 20771 \\ Received 1999 December 12; accepted 2000 September 18
}

\begin{abstract}
Intensities of the extreme-ultraviolet (EUV) spectral lines were measured as a function of radius off the solar limb by two flights of the Goddard's Solar Extreme-Ultraviolet Rocket Telescope and Spectrograph (SERTS) for three quiet-Sun regions. Density scale heights were determined for the different spectral lines. Limits on the filling factor were determined. In the one case where an upper limit was determined it was much less than unity. Coronal heating above 1.15 solar radii is required for all three regions studied. For reasonable filling factors, local heating is needed.
\end{abstract}

Subject headings: Sun: corona - Sun: UV radiation

\section{INTRODUCTION}

In the corona, the magnetic field pressure is strong compared to the gas pressure $(\beta \ll 1)$. As a result relatively large cross-field gradients in the electron density can be balanced by relatively small gradients in the background magnetic field strength. Also in low- $\beta$ plasma, electron heat conduction is primarily along the magnetic field lines, with negligible conduction across the field lines (Spitzer 1962). So the magnetic field in the corona isolates adjacent magnetic structures allowing them to have different temperatures and densities. For a more in-depth discussion than is given here, see Giovanelli (1984).

While the magnetic field pressure controls the physical morphology of a given coronal structure, the magnitude of the heating occurring in a structure controls the radiative flux (Rosner, Tucker, \& Vaiana 1978). If we consider a loop initially in thermal equilibrium and then we allow the heating rate to increase, the temperature will rise, causing the temperature gradient parallel to the magnetic field to increase, which will increase the conductive heat flux to the transition region. This will cause additional plasma to be heated and rise into the corona, increasing the coronal density in the structure. A new equilibrium is reached when the total radiative and conductive flux is equal to the heating rate. When the heating rate decreases, the plasma will cool radiatively and return to the transition region. The density of the coronal structure will then decrease, lowering the radiative losses so a new equilibrium can be reached.

In spectrometers, variations can be detected on scales smaller than the spatial resolution of the observing instrument. Using line-ratio diagnostics we can sometimes determine the electron density and temperature (Feldman \& Doschek 1977). We typically can then estimate the total emission assuming uniform filling along a given line of sight and compare it to the observed emission. We find that the observed emission is smaller than the total emission, so only a fraction of the volume is filled with plasma. This has given rise to the concept of the filling factor, which is defined as the fraction of the volume filled with corona plasma emit-

\footnotetext{
${ }^{1}$ Marshall Space Flight Center/NASA, SD 50, Huntsville, AL 35812.
}

ting at the wavelengths observed. If the filling factor is unity, then the volume of corona plasma observed has a constant plasma density. If the filling factor is much less than unity, then only a fraction of the volume is responsible for the emission observed.

At present, the precise nature of the mechanism responsible for heating the coronal plasma has not yet been determined. The two primary models are (1) the dissipation of waves propagated along the coronal magnetic field lines, or (2) the dissipation of currents conducted along these field lines. Also, different hybrid models exist. See Zirker (1993) for a recent survey of coronal heating theories. In this paper, we use SERTS observations of the low corona at the solar limb to derive conditions that any successful heating theory must satisfy.

From the exponential decrease of the spectral line intensity with height, we can determine the density scale height, which is used to estimate the plasma temperature. An independent measure of plasma temperature is obtained from the observed ratios of emission line intensities from different ionization states of a single element. The scale-height temperature is then compared to the line-ratio temperature. Generally, we find that the line-ratio temperature and scaleheight temperature are in agreement, indicating that the bulk of the plasma is thermal.

By determining the radial dependence of the temperature and the emission measure we can determine observationally whether local heating is needed. This is done by first estimating the divergence of the heat flux from the radial temperature gradient. This is compared to the radiative flux, which is determined from the emission measure, and the electron temperature using emissivities from Cook et al (1989). The difference between these two quantities indicates whether local heating is needed in a steady state condition. We find that, typically, local heating is required in the region $r<1.2 R_{\odot}$ for all the cases observed by SERTS.

\section{OBSERVATIONS}

\subsection{Instrument}

The Solar Extreme-Ultraviolet Rocket Telescope and Spectrograph (SERTS) instrument obtains both images and spectra of solar coronal regions recorded on film, from 235 


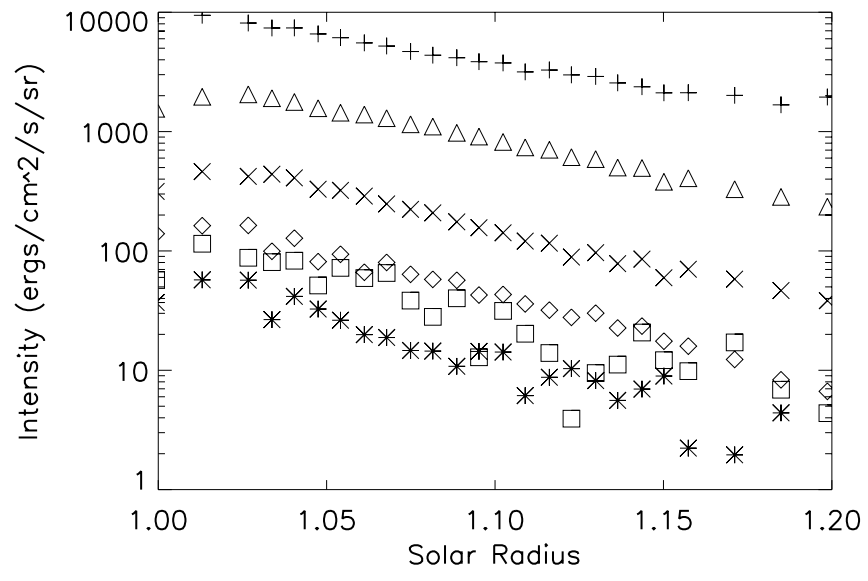

FIG. $1 a$

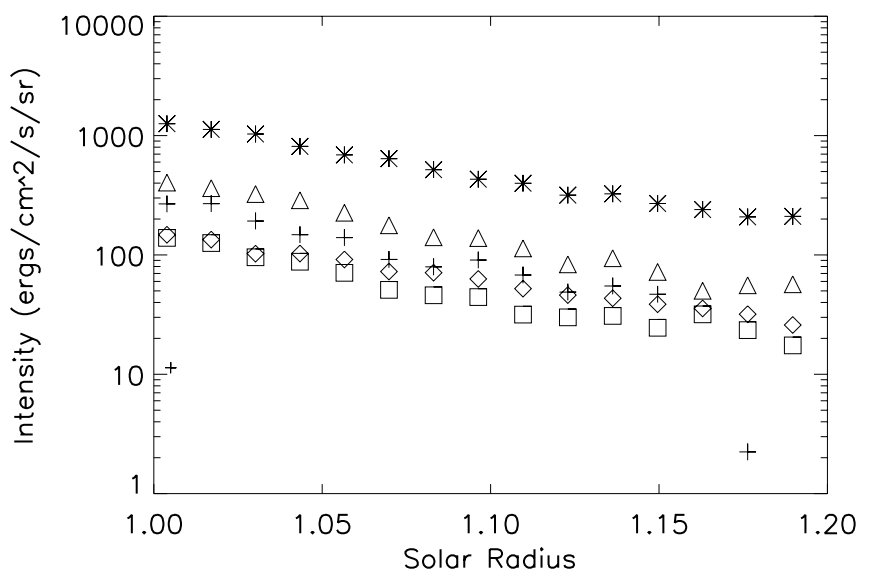

FIG. $1 b$

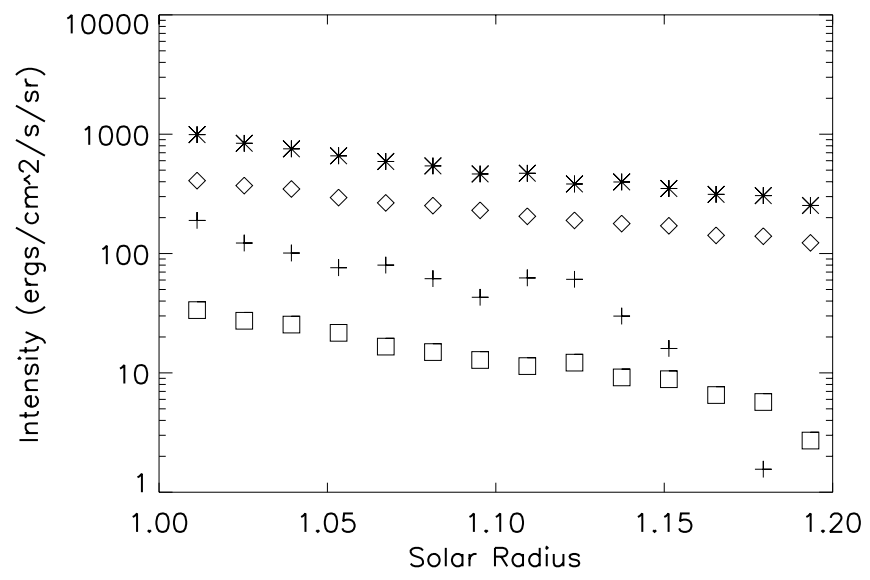

FIG. 1c

Fig. 1.-Intensity scale heights for the three regions. From the radial dependence of the spectral line's intensity, the density scale height can be estimated taking into account geometric effects and temperature effects for SERTS 1991 southern edge $(a)$, SERTS 1989 southern edge $(b)$ and SERTS 1989 northern edge $(c)$. For Fig. $1 a$, the spectral-line intensities shown are Fe XVI $\lambda 361$ (plus signs), Fe XIV $\lambda 334$ (crosses), Fe XIII $\lambda 360$ (squares), Cr XIII $\lambda 328$ (asterisks), Si XI $\lambda 303$ (triangles), and $\mathrm{Al} X \lambda 332$ (diamonds). The spectral lines with the weakest intensities have the largest signal to noise. For Fig. 1b, the spectral-line intensities shown are Fe XVI $\lambda 361$ (diamonds), Fe XV $\lambda 284$ (asterisks), Fe XIV $\lambda 334$ (squares), Fe XIV $\lambda 274$ (plus signs), and Si XI $\lambda 303$ (triangles). For Fig. 1 c, the spectral-line intensities shown are Fe XVI $\lambda 361$ (triangles), Fe XV $\lambda 284$ (asterisks), Fe XV $\lambda 417$ (squares), and Fe XIV $\lambda 274$ (plus signs). Beyond $1.15 R_{\odot}$ the Fe XIV $\lambda 274$ (plus signs) intensity is unreliable for Figs. $1 b$ and $1 c$.

to $450 \AA$ in first order, and from 170 to $225 \AA$ in second order. The SERTS 1989 instrument is described in Neupert et al. (1992), with modifications described by Thomas et al. (1991). The pointing positions were determined (Brosius et al. 1993) and the images were digitized and calibrated (Thompson et. al. 1993; Thomas \& Neupert 1994) with an estimated factor of 2 uncertainty in the absolute calibration, which will only affect the absolute values of the emission measure, not the relative values. The film data was digitized and merged into $150 \times 150 \mu$ m pixels $\left(13^{\prime \prime} \times 13^{\prime \prime}\right)$, which are used for the analysis in this paper.

\subsection{Spectrograph Data Format}

The SERTS instrument obtains both slit spectra and spectroheliograms (see Falconer, Davila, \& Thomas 1997, Fig. 4). The middle region provides a spatially resolved high-resolution spectrum of a $2^{\prime \prime} \times 5^{\prime}$ portion of the solar disk. The upper and lower regions are produced by the instrument lobes (see Falconer et al. 1997, Fig. 5) where the direction parallel to the slit is still purely spatial but the direction perpendicular to the slit contains both spatial and wavelength information. Because of the domination of the spectrum in the EUV by strong spectral lines and the nature of our entrance aperture, we often can separate spatial and wavelength dependence. This separation is easiest for bright spectral lines that have no other bright overlapping images. In such cases, the presentation can be considered a spectroheliogram. The lobe images for both SERTS 1989 and SERTS 1991 flights contain observations to heights of 1.15 $R_{\odot}$, which we will analyze for this paper.

\subsection{Determination of Spectral Line Intensity}

We can determine the intensity of the spectral line by measuring the increase in brightness across the edges of the lobes. This is done by subtracting the background intensity from the intensity at the edge of the lobe to determine the intensity of the spectral line as a function of radius (See Falconer et al. 1997, Fig. 4). The larger the separation between the position where we determine the background and the position where we measure the intensity of the lobe, the greater the potential uncertainty is in the determination of the spectral line intensity. So, we limit ourselves to determining the intensity only at the edge of the lobe, which will give us the intensity as a function of height. The systematic 
uncertainty in the spectral line intensity determined by this method is around $15 \%$. This systematic uncertainty would effect the overall intensity of the spectral line and not the relative intensities of the different pixels. The systematic uncertainty is larger than the variation in the intensity of the spectral lines versus radius due to noise except for very weak spectral lines.

\subsection{Line Selection and Determination of Spectral Line Intensity}

We required that for a spectral line to be included in our analysis, it must meet the following conditions:

1. The line should be strong compared to the background.

2. Adjacent lines must be spectrally resolved.

3. The emission mechanism must be well understood

4. Any overlapping images should be weak.

Observations from both lobe edges of the SERTS 1989 flight were usable for this analysis. They were both on the western limb and one set (the shorter wavelength edge) was of a position to the south of the other. So the two data sets will be called SERTS 1989 southern edge and SERTS 1989 northern edge. SERTS 1989 southern edge was found to have five spectral lines that satisfied the selection criteria: Si XI $\lambda 303$, Fe XIV $\lambda 274, \mathrm{Fe}$ XIV $\lambda 334, \mathrm{Fe}$ XV $\lambda 284$, and Fe XVI $\lambda 361$. SERTS 1989 northern edge had Fe XIV $\lambda 274, \mathrm{Fe}$ XV $\lambda 284, \mathrm{Fe} \mathrm{xv} \lambda 417$, and Fe $\mathrm{xvI} \lambda 335$. The intensity as a function of height for the different spectral lines is shown in Figures $1 b$ and $1 c$.

Observations from only the southern lobe edge of the SERTS 1991 flight were found to be usable for this analysis. SERTS 1991 southern edge was found to have six spectral lines that satisfied the selection criteria: $\mathrm{Al} \times \lambda 332, \mathrm{Cr}$ XIII $\lambda 328, \mathrm{Si}$ XI $\lambda 303, \mathrm{Fe}$ XIII $\lambda 360, \mathrm{Fe}$ XIV $\lambda 334$, and Fe XVI $\lambda 361$. (The Fe XIII $\lambda 360$ line was called 359.63 in Falconer et al. (1997) owing to the need to distinguish it from the Fe XIII 2359.84 spectral line). This data set is called SERTS 1991 southern edge. The reasons for the changes in selected lines between the two flights were the wider wavelength coverage in SERTS 1989 flight, the increased efficiency, and intensity of emission in SERTS 1991 flight. The intensity as a function of height for the different spectral lines is shown in Figure 1a. As was discussed in Falconer et al., the Fe XIII $\lambda 360$ line had an additional complication of a nearby lobe that overlaps the region, which to correct for we assign an uncertainty of $25 \%$ for this line instead of the normal $15 \%$.
A combination of observations from two different pointing positions covered the range from $1.0 R_{\odot}$ to $1.2 R_{\odot}$.

\subsection{Observations}

All three data sets come from the western solar limb, so we can determine the sunspot locations behind the solar limb by examining the observations from a quarter of a rotation earlier. In Falconer et al. (1997), we classified the two SERTS 1989 observations as quiet Sun. The SERTS 1991 southern edge had no active regions on the solar limb but had a hot loop near the southern edge of the lobe, and also a number of foreground and background active regions, so these observations are classified as coming from a more active quiet-Sun region, since more emission from the active regions is seen along the line of sight.

\section{DENSITY SCALE HEIGHT}

Figure 1 shows the radial dependence of the intensity of several spectral lines. Guhathakurta et al. (1992) derived the radial dependence of intensity for a line-of-sight off the solar limb for a spherical atmosphere, with a hydrostatic atmosphere as

$$
I(r)=R_{\odot}\left(\frac{2 \pi H_{s}}{\delta}\right)^{1 / 2} N_{e o}^{\delta} E_{\delta} r^{1.5} \exp \left[\frac{-\delta}{H_{s}}\left(\frac{1}{r_{0}}-\frac{1}{r}\right)\right],
$$

$H_{s}$ is the density scale height, $r_{o}$ is the altitude at which $N_{e}(r)$ equals $N_{e o}, \delta$ is a parameter that can range from 1 to 2 depending on if the spectral line is dominated by resonance scattering $(\delta=1)$ or electron collision excitation $(\delta=2)$, and $E_{\delta}$ is the total emissivity, which combines both resonance scattering and collisional excitation. $H_{s}, r$, and $r_{o}$ are all in units of solar radius $\left(R_{\odot}=6.96 \times 10^{10} \mathrm{~cm}\right)$. For cases where collisional excitation dominates, $E_{\delta}$ would just be the spectral lines contribution function $Q\left(T_{e}\right)$, which is itself primarily dependent on the electron temperature, $T_{e}$ (that $Q$, for some spectral lines, also have a density dependence is ignored for this analysis because of added complications). As was done in Falconer et al. (1997), the contribution functions used for the different spectral lines comes from using Arnaud \& Rothenflug (1985) ionization balances and emissivities from Monsignori-Fossi \& Landini (1995). We calculate $E_{\delta}$ for each spectral line as a function of radius, assuming electron collision dominates, using the parameterized function of the temperature given in Falconer et al. (1997) that are listed in Table 1, and assuming $H_{s}=0.1 R_{\odot}$ for the calculation of $Q$. Our results are not sensitive to

TABLE 1

LiNe-RATio Temperatures AND EmisSion MeASURES

\begin{tabular}{ccccc}
\hline \hline Edge & $\begin{array}{c}\text { Range } \\
\left(R_{\odot}\right)\end{array}$ & $\begin{array}{c}T_{o}^{\mathrm{a}, \mathrm{b}} \\
\left(\times 10^{6}\right)\end{array}$ & $\begin{array}{c}a^{\mathrm{a}, \mathrm{b}} \\
\left(\times 10^{6}\right)\end{array}$ & Emission Measure at $1.05 R_{\odot}^{\mathrm{c}, \mathrm{d}} \mathrm{cm}^{-5}$ \\
\hline SERTS 1991 South ...... & $1.05-1.19$ & $1.87 \pm 0.13$ & $0.51 \pm 0.22$ & $8.7 \times 10^{27}(1.2)^{\mathrm{e}}$ \\
SERTS 1989 South ...... & $1.02-1.15$ & $1.75 \pm 0.12$ & $0.71 \pm 0.30$ & $3.6 \times 10^{27}(1.5)$ \\
SERTS 1989 North ...... & $1.02-1.15$ & $1.97 \pm 0.15$ & $1.13 \pm 0.49$ & $3.2 \times 10^{27}(1.8)$ \\
\hline
\end{tabular}

\footnotetext{
a $T_{e}=T_{o}+a(r-1)$.

b Uncertainties in $T_{0}$, include the fitting parameter, the calibration uncertainty, and the theoretical uncertainties.

c Assuming (Meyer 1985) Elemental Abundance for iron.

d The emission measure obtained for Fe XIV $\lambda 334$ is reported except for SERTS 1989 North where FE XV $\lambda 417$ is reported.

e The error factor ("within a factor of ...") is a combination of the instrument uncertainty and the uncertainty due to the line-ratio temperature. It does not include the absolute flux uncertainty of a factor of 2 .
} 
what $H_{s}$ is assumed for the calculation of $Q$, for example if we instead set $H_{s}=0.2 R_{\odot}$ we get results within $1 \%$ of those for $H_{s}=0.1 R_{\odot}$. Rearranging equation (1) to

$$
\ln \left\{\frac{I(r)}{Q\left[T_{e}(r)\right] r^{1.5}}\right\}=A-\frac{\delta}{H_{s}}\left(\frac{1}{r_{0}}-\frac{1}{r}\right),
$$

and using a first order polynomial fit with respect to $\left(1 / r_{o}\right)$ $-(1 / r)$, we get $H_{s} / \delta$. These are tabulated in Table 2 , as $H_{s} /(\delta / 2)$ since for most lines $\delta$ will be equal to 2 . The quoted uncertainty is from the fit with the condition that the uncertainty cannot be less than $10 \%$ because of theoretical uncertainties in $Q$. For any particular region, $H_{s} /(\delta / 2)$ for most spectral lines are similar, with only one or two spectral lines having results significantly larger than the others. An emissivity-corrected density scale height is chosen for each observed region from the spectral lines which have similar $H_{s} /(\delta / 2)$, assuming $\delta=2$. The value of the emissivitycorrected scale height is given in Table 3 and is used to calculate $\delta$ for each spectral line in Table 2 .

There are two cases where $\delta$ is significant less than 2 ( $\mathrm{Si} \mathrm{XI}$ 2303, SERTS 1989; and Fe XV $\lambda 284$, SERTS 1991 northern edge), indicating that these spectral lines are likely influenced by both electron collision excitation and resonance scattering due to a strong underlying radiation. The exact importance of these two sources cannot be modeled, since the light that is resonantly scattered would be coming from the limb.

In Table 3 we compare the emissivity-corrected density scale height, the scale-height temperature, and the line-ratio temperature at $1.1 R_{\odot}$. In these results, the scale-height temperatures, $T_{s}\left(H_{s}=0.0729 \times 10^{-6} T_{s}\right.$, Guhathakurta et al. 1992) and the line-ratio temperatures are similar, but they can differ from each other by half a million degrees. In two of the cases, the line-ratio temperature is higher, while in the third the scale-height temperature is larger. The primary reason for these differences is probably due to the failure of the assumption of spherical symmetry. Secondary reasons for these differences can be turbulence, changes in the filling factor, nonhydrostatic conditions such as flows, and divergence of the magnetic field; all are nonthermal effects that can change the density scale height. It should be pointed out that except for determining $Q$, the two temperatures were independently determined.

TABLE 2

Temperature from Scale Height

\begin{tabular}{ccccc}
\hline \hline Ion $\lambda$ & Temperature of Maximum Abundance ${ }^{\mathrm{a}}$ & $\begin{array}{c}\text { Range }^{\mathrm{b}} \\
\left(R_{\odot}\right)\end{array}$ & $\begin{array}{c}2 H_{s} /(\delta / 2) \\
\left(R_{\odot}\right)\end{array}$ & $\delta$ \\
\hline Fe XVI $\lambda 361 \ldots \ldots$ & SERTS 1991 Southern Edge & & \\
Fe XIV $\lambda 334 \ldots \ldots$ & $2.57 \mathrm{e} 6$ & $1.05-1.20$ & $0.10 \pm 0.01$ & $1.9 \pm 0.2$ \\
Fe XIII $\lambda 360 \ldots \ldots$ & $1.82 \mathrm{e} 6$ & $1.05-1.20$ & $0.10 \pm 0.01$ & $1.9 \pm 0.2$ \\
Si XI $\lambda 303 \ldots \ldots \ldots$ & $1.58 \mathrm{e} 6$ & $1.05-1.14$ & $0.09 \pm 0.01$ & $2.0 \pm 0.2$ \\
Cr XIII $\lambda 328 \ldots \ldots$ & $1.58 \mathrm{e} 6$ & $1.05-1.20$ & $0.12 \pm 0.01$ & $1.5 \pm 0.2$ \\
Al x $\lambda 332 \ldots \ldots \ldots$ & $1.55 \mathrm{e} 6$ & $1.05-1.18$ & $0.10 \pm 0.01$ & $1.9 \pm 0.2$ \\
& $1.26 \mathrm{e} 6$ & $1.05-1.20$ & $0.09 \pm 0.01$ & $2.1 \pm 0.2$ \\
\hline & SERTS 1989 Southern Edge ${ }^{\mathrm{d}}$ & & \\
\hline Fe XVI $\lambda 361 \ldots \ldots$ & $2.57 \mathrm{e} 6$ & $1.05-1.15$ & $0.12 \pm 0.01$ & $2.0 \pm 0.2$ \\
Fe XV $\lambda 284 \ldots \ldots$ & $2.09 \mathrm{e} 6$ & $1.05-1.15$ & $0.12 \pm 0.01$ & $2.0 \pm 0.2$ \\
Fe XIV $\lambda 334 \ldots \ldots$ & $1.82 \mathrm{e} 6$ & $1.05-1.14$ & $0.13 \pm 0.01$ & $1.9 \pm 0.2$ \\
Fe XIV $\lambda 274 \ldots \ldots$ & $1.82 \mathrm{e} 6$ & $1.05-1.14$ & $0.13 \pm 0.01$ & $1.9 \pm 0.2$ \\
Si XI $\lambda 303 \ldots \ldots \ldots$ & $1.58 \mathrm{e} 6$ & $1.05-1.15$ & $0.12 \pm 0.01$ & $1.9 \pm 0.2$ \\
\hline & SERTS 1989 Northern Edge ${ }^{\mathrm{e}}$ & & \\
\hline Fe XVI $\lambda 335 \ldots \ldots$ & $2.57 \mathrm{e} 6$ & $1.05-1.14$ & $0.18 \pm 0.02$ & $1.9 \pm 0.2$ \\
Fe XV $\lambda 284 \ldots \ldots$ & $2.09 \mathrm{e} 6$ & $1.05-1.15$ & $0.21 \pm 0.02$ & $1.6 \pm 0.2$ \\
Fe XV $\lambda 417 \ldots \ldots$ & $2.09 \mathrm{e} 6$ & $1.05-1.15$ & $0.16 \pm 0.02$ & $2.1 \pm 0.2$ \\
Fe XIV $\lambda 274 \ldots \ldots$ & $1.82 \mathrm{e} 6$ & $1.05-1.13$ & $0.19 \pm 0.05$ & $1.8 \pm 0.4$ \\
\hline
\end{tabular}

a The temperatures of maximum abundance was determined using Arnaud \& Raymond 1992 for iron, Arnaud \& Rothenflug (1985) for aluminum and silicon, and Monsignori-Fossi (private communication) for chromium.

$\mathrm{b}$ The range was reduce for certain lines due to low signal to noise.

c $\delta$ was caculated assuming a density scale height of $0.095 R_{\odot}$.

d $\delta$ was caculated assuming a density scale height of $0.12 R_{\odot}$.

e $\delta$ was caculated assuming a density scale height of $0.17 R_{\odot}$.

TABLE 3

Comparison SCAle Height vs. Line-Ratio Temperature

\begin{tabular}{lccc}
\hline \hline Observation ............. & Emissivity-Corrected Density Scale Height & Scale-Height Temperature & Line-Ratio Temperature $^{\mathrm{a}}$ \\
\hline SERTS 1991 South ...... & 0.095 & $1.3 \mathrm{e} 6$ & $1.9 \mathrm{e} 6$ \\
SERTS 1989 South ...... & 0.12 & $1.6 \mathrm{e} 6$ & $1.8 \mathrm{e} 6$ \\
SERTS 1989 North ...... & 0.17 & $2.3 \mathrm{e} 6$ & $2.1 \mathrm{e} 6$ \\
\hline
\end{tabular}

a The line-ratio temperature at $1.1 R_{\odot}$. 


\section{FILLING FACTOR AND MINIMUM ELECTRON DENSITY}

Since the emission measure is determined along a line of sight, the small-scale structures are averaged in this observed volume. While the line-ratio density determines the weighted average density of the emitting structures, the emitting structure may not completely fill the potentially observed volume. The filling factor is the volume of the emitting structures divided by the observed volume. If the filling factor is less than unity, then only a fraction of the observed volume is filled with plasma emitting in the observed wavelength. If the filling factor is 1 , the observed volume is uniformly filled.

The average of the electron density squared, $\overline{N_{e}^{2}}$, is related to the emission measure by

$$
\overline{N_{e}^{2}}=\frac{\eta}{f L},
$$

where $\eta$ is the emission measure, $f$ is the filling factor, and $L$ is the effective line of sight. From Guhathakurta et al. (1992), assuming a spherical hydrostatic atmosphere

$$
L=R_{\odot}\left(\frac{2 \pi H_{s}}{\delta}\right)^{0.5} r^{1.5}
$$

where $r$ and $H_{s}$ are both in solar radii units. The minimum electron density necessary $(f=1)$ to explain the observed emission measure will then be

$$
\left(N_{e}\right)_{\min } \equiv \sqrt{\frac{\eta(r)}{L(r)}} .
$$

The minimum electron density at $1.05 R_{\odot}$ is given in Table 4. The emission measures, $\eta$, were determined in Falconer et al. (1997) and are given in Table 1, assuming Meyer's (1985) elemental abundances. The limits on the filling factor can be determined by comparing the minimum electron density and the limits on line-ratio density given in Table 4 from Falconer et al. (1997). The line-ratio density is an average density along the line of sight and not the density at a single radial position. For an exponential atmosphere with a density scale height of less than $0.2 R_{\odot}$, the line-ratio density times $\sqrt{2}$ would be the electron density at the point along a line of sight closest to the center of the Sun. Substituting equation (5) into equation (3), the filling factor would then be

$$
f=\frac{1}{2}\left[\frac{\left(N_{e}\right)_{\min }}{\overline{N_{e}}}\right]^{2},
$$

where $\overline{N_{e}}$ is the line-of-sight density determined from the line-ratio density. The limits on the filling factor are given in the last column of Table 4. If we used instead Meyer (1996) elemental abundances (where low FIP elements are enhanced relative to hydrogen), the upper and lower limits to the filling factor would be a factor of 3.38 smaller.

Since the line-ratio density for SERTS 1991 southern edge below $1.1 R_{\odot}$ was determined to be between $10^{9}$ and $10^{10} \mathrm{~cm}^{-3}$, this implies a filling factor between $4 \times 10^{-4}$ to $4 \times 10^{-2}$, using Meyer's (1985) elemental abundances. The filling factors for both SERTS 1989 southern and northern edges need to be greater than $7 \times 10^{-5}$ because of an upper limit on the line-ratio density of $10^{10} \mathrm{~cm}^{-3}$.

\section{HEATING}

In Falconer et al. (1997), we found that the line-ratio temperature increased with radius so heating above the observed height range $\left(1.15 R_{\odot}\right.$ for SERTS 1989 and $1.2 R_{\odot}$ for SERTS 1991) is needed. This radial increase in the lineratio temperature also implies that heat would be conducted toward the transition region. Could this conductive heat flux be the sole source of energy for the radiative flux or is local heating required?

To determine if local heating is required, one has to compare the energy deposited by heat conduction, to the energy radiated away. A steady state energy balance implies that

$$
H=\nabla \cdot F_{c}+F_{r},
$$

where $H$ is the local heating rate, $F_{c}$ is the conductive flux, and $F_{r}$ is the radiative losses. From the radial dependence of the line-ratio temperatures, $\nabla \cdot F_{c}$ is estimated, which then can be compared to $F_{r}$ to determine if local heating is needed.

At coronal temperatures, electron heat conduction dominates the conducting flux according to Spitzer (1962) along magnetic field lines. It is

$$
F_{c}=10^{-6} T^{2.5} \frac{d T}{d z},
$$

for a Maxwellian particle distribution where $T$ is the temperature and $z$ is the distance along the magnetic field line. Cross magnetic field heat conduction is negligible, for example for $N_{e}=10^{9} \mathrm{~cm}^{-3}, B=10$ gauss, and $T=2 \times 10^{6}$ $\mathrm{K}$, the ratio of the conductive flux across magnetic field lines to along the field line is $10^{-10}$.

One can obtain an upper limit on the divergence of the conductive flux in the following way. We assume the conductive flux for $r_{1} \leq r \leq r_{2}$ has a functional dependence of

$$
F_{c}(r)=F_{o}-\left(r_{2}-r\right) F_{1}+\ldots,
$$

where we keep only the linear terms, with $F_{o}$ being the heat flowing along the field line at altitude $r_{2}$, and $F_{1}$ is the

TABLE 4

Minimum Electron Density and Filling Factor at $1.05 R_{\odot}$

\begin{tabular}{cccr}
\hline \hline Data Set & Minimum Electron Density ${ }^{\mathrm{a}, \mathrm{b}}$ & Line-Ratio Density & \multicolumn{1}{c}{ Filling Factor $^{\mathrm{a}, \mathrm{b}}$} \\
\hline SERTS 1991 South ...... & $4.4 \times 10^{8}(1.09)$ & $10^{9}-10^{10}$ & $0.1-0.001(1.17)$ \\
SERTS 1989 South ..... & $2.8 \times 10^{8}(1.26)$ & $<10^{10}$ & $>4.0 \times 10^{-4}(1.51)$ \\
SERTS 1989 North ...... & $2.7 \times 10^{8}(1.40)$ & $<10^{10}$ & $>3.5 \times 10^{-4}(1.80)$ \\
\hline
\end{tabular}

\footnotetext{
${ }^{a}$ For Meyer 1985 elemental abundances.

${ }^{b}$ A systematic uncertainty of a factor of 2.0 in the filling factor, and a factor of 1.4 for the minimum electron density exist because of the absolute calibration (see Falconer et al. 1997).
} 
divergence of the conductive flux. If we set

$$
F_{c}\left(r_{1}\right)=b F_{o},
$$

where $b$ is the ratio of the heat conducting at $r_{2}$ to that at $r_{1}$, this makes

$$
F_{1}=\frac{(1-b) F_{o}}{\Delta r},
$$

where $\Delta r=\left(r_{2}-r_{1}\right)$.

The temperature as a function of radius is given by

$$
\frac{2 \times 10^{-6}}{7}\left(T_{2}^{3.5}-T^{3.5}\right)=F_{o}\left(r_{2}-r\right)-\left(r_{2}-r\right)^{2} F_{1} / 2 .
$$

This relationship is derived from integrating equation (8) and substituting in the linear version of equation (9), where $F_{o}$, and $F_{1}$ are both functions of $b$, and dependent on the temperatures at $r_{1}$, and $r_{2}$, for which we will use the linear fit to the line-ratio temperature given in Table 1 . The functional form of $F_{o}$ is

$$
F_{o}=\frac{4 \times 10^{-6}\left(T_{2}^{3.5}-T_{1}^{3.5}\right)}{7 \Delta r(1+b)},
$$

with $F_{1}$ related to $F_{\mathrm{o}}$ by equation (11). Figure 2 shows for one region, the line-ratio temperature, as well as the temperatures from equation (12), assuming either $b=0$ (dotted line), where all the heat conducted is dissipated locally, or $b=1$ (solid line), where no heat is locally dissipated and all the energy radiated has to be from local heating. As can be seen, $b=1$ fits the data better than $b=0$, but $b=0$ cannot be ruled out. Table 5 gives $F_{o}, F_{1}$ for the case of $b=1$, where the uncertainty in $F_{1}$ is set by using $b=0$.

The radiation per unit volume, $F_{r}$, is estimated from the observations by

$$
F_{r}=\varepsilon_{T}(T) \overline{N_{e}^{2}}\left(\operatorname{ergs~cm}{ }^{-3} \mathrm{~s}^{-1}\right),
$$

where $\varepsilon_{T}$ is the total radiative loss function, which is a function of the temperature and the elemental abundances (Cook et al. 1989) using Meyer's (1985) elemental abundances. Because of how iron spectral lines dominate the emission over temperature ranges observed and that our measurements used iron spectral lines to get the emission measure and line-ratio temperatures, different assumptions about the elemental abundances have small effect on the total radiative flux. Substituting equation (3) for $N_{e}^{2}$, equation (14) becomes

$$
F_{r}=\varepsilon_{T} \eta(r) / f L(r) .
$$

The values of $F_{r}$ are tabulated in Table 5 at a height of 1.05 , as a function of $f$, the filling factor. Comparing the upper

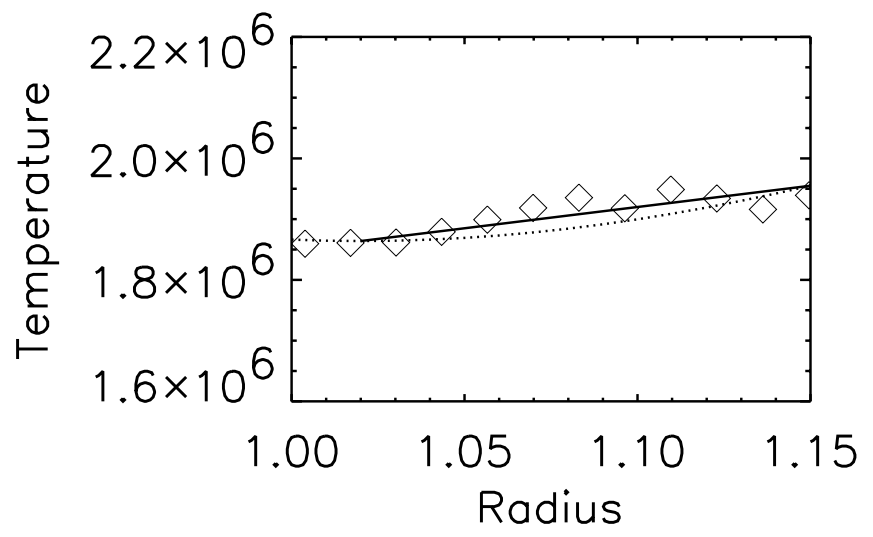

Fig. 2.-Radial temperature dependence for two models of the conductive heat flux. The diamonds show the observed line-ratio temperature for Fe XIV $\lambda 334 / F e$ XVI $\lambda 361$, SERTS 1989 southern side. There is a roughly $150,000 \mathrm{~K}$ systematic uncertainty in the line-ratio temperature due to absolute instrument calibration and uncertainty in the theoretical line ratio. This uncertainty though would effect all the line-ratio temperature measurements in the same direction by nearly equal amounts. The radial temperature profiles shown are for $b=1$ (solid line) and $(b=0)$ (dotted line). The two models are constrained to fit the line-ratio temperatures at 1.02 and $1.15 R_{\odot}$. The curve for $b=1$ and no divergence of the conductive heat flux fits the observations better, but $b=0$, cannot be ruled out.

limits on the divergence of the heat flux to the radiative flux, assuming a filling factor of unity, SERTS 1991 needs local heating. For more likely filling factors of less than 0.1 , as was measured for SERTS 1991, all three cases need local heating. Remember that Figure 2 shows that zero divergence of the conductive flux fits the line-ratio temperatures better than our upper limit of all the conductive flux deposited into the range observed, indicating that local heating is probably needed in the third cases.

\section{DISCUSSION AND CONCLUSIONS}

The line-ratio temperatures and scale-height temperatures agree to within approximately $25 \%$ (Table 3 ). The scale-height temperature was derived from the radial dependence of the intensity. The line-ratio temperature was determined from the ratio of the intensities of different spectral lines. These two temperature measurements were nearly independent. Since the line-ratio temperature requires fewer assumptions and can be used to determine the radial temperature dependence, it is the preferred method for determining the coronal temperature. Reliable determination of the line-ratio temperature requires many spectral lines from different ionization stages of the same element, where none of the lines is strongly affected by resonance scattering.

An upper limit was determined for the filling factor for SERTS 1991 southern edge of 0.1 . This means that less than $10 \%$ of the volume observed dominates the emission of the

TABLE 5

Comparison of Radiation to the Divergence of the Heat Flux

\begin{tabular}{ccccr}
\hline \hline Observation & $\begin{array}{c}\text { Range } \\
\left(R_{\odot}\right)\end{array}$ & $\begin{array}{c}F_{\mathrm{o}} \\
\left(\mathrm{ergs} \mathrm{cm}^{-2} \mathrm{~s}^{-1}\right)\end{array}$ & $\begin{array}{c}F_{1} \\
\left(\mathrm{ergs} \mathrm{cm}^{-3} \mathrm{~s}^{-1}\right)\end{array}$ & $\begin{array}{c}F_{r} \\
\left(\mathrm{ergs} \mathrm{cm}^{-3} \mathrm{~s}^{-1}\right)\end{array}$ \\
\hline SERTS 1991 South ...... & $1.1-1.2$ & $4 \times 10^{4}$ & $0 \pm 9 \times 10^{-6}$ & $16.0 \times 10^{-6} / f(1.22)$ \\
SERTS 1989 South ....... & $1.02-1.15$ & $5 \times 10^{4}$ & $0 \pm 10 \times 10^{-6}$ & $7.8 \times 10^{-6} / f(1.73)$ \\
SERTS 1989 North ...... & $1.02-1.15$ & $10 \times 10^{4}$ & $0 \pm 30 \times 10^{-6}$ & $4.1 \times 10^{-6} / f(2.13)$ \\
\hline
\end{tabular}

NoTE. $-F_{r}$ determined at $1.05 R_{\odot} . F_{o}, F_{1}$, and $F_{r}$ are respectively the constant part of the heat conduction, divergence of the heat conduction, and the radiative power. The radiative power needs to be divided by the filling factor, $f$, which for SERTS 1991 is less than 0.1 . 
EUV spectral lines. What could be in the other $90 \%$ or greater of the volume? It has to be something that does not emit strongly in the EUV spectral lines observed. It could be low-density plasma since the emission from electron collision goes as the density square; if the density was only one-tenth of that in the emitting structures, the emission would drop by a factor of 100 for the same volume. Also, if the plasma was cooler, say $1 \times 10^{6} \mathrm{~K}$, it would not emit strongly in the spectral lines observed. If the temperature was several million degrees, the plasma would only emit weakly in the observed spectral lines. These low filling factors from SERTS observations are not surprising considering the filamentary structures seen in higher resolution TRACE images.

In Table 1, all the line-ratio temperatures increase with height. Neupert et al. (1998) and Aschwanden et al. (1999) have also found individual loops observed by either $\mathrm{SOHO}$ / EIT and TRACE have temperatures increasing with height. To supply the heat that would be conducted downward and lost from the plasma as radiation, a source of heat is required at some location above the maximum observed height $\left(1.15 R_{\odot}\right.$ for SERTS 1989 flight and $1.2 R_{\odot}$ for SERTS 1991 flight).

The upper limits on the divergence of the heat flux is less than the radiative flux, and therefore local heating is needed for SERTS 1991 below $1.15 R_{\odot}$ for a filling factor of unity. Assuming a more likely filling factor of 0.1 or less as was measured for SERTS 1991, all three cases would need local heating. Since the filling factor is less than 1 , the two constraints on heating just mentioned only apply to the magnetic flux tubes that have coronal plasma which we observed. The other flux tubes could have different heating requirements. Other researchers using SOHO/EIT (Aschwanden et al. 1999) and TRACE (Neupert et al. 1998; Lenz et al. 1999) observations have shown that individual loops, also need local heating. The temperatures derived in these other observations were lower than the ones derived from SERTS, this could be due to the solar cycle variations (The SOHO/EIT observations were from 1996, while the TRACE observations were from 1998), or more probably the different wavelengths sampling different fractions of the corona (their observations were using the $\mathrm{Fe} \mathrm{Ix} / \mathrm{x}$ and $\mathrm{Fe} \mathrm{XII}$ filters), and the filled flux tubes having a range of temperatures.

In the introduction, we mentioned that magnetic topology controls the solar corona. Except for the hot loops observed by the SERTS 1991 flight, the quiet-Sun corona appears relatively uniform with only a radial dependence. The upper limit of the filling factor of less than 1, for SERTS 1991 southern edge, indicates unseen structures exist. The relatively uniform nature of the background corona suggests that many plasma filled magnetic flux tubes exist along the line of sight. Later, high resolution TRACE observations are showing filamentary structures, helping to confirm filling factors of less than 1 .

Such a filamentary corona complicates the comparison of temperatures or densities derived from different instruments as follows. This all suggests that the quiet corona is filamentary, with different filaments filled with plasma at different temperatures and densities. Instruments like SERTS, EIT, and quite possibly even the higher resolution TRACE average over a series of these unresolved filaments, weighted by the spectral lines, or spectral bands that the instrument observes, with the SERTS observation sampling hotter plasma compared to the TRACE and EIT observations. The resulting averages result in the line-of-sight emission measures, electron temperatures, electron densities, and filling factors along with the radial dependence of the temperature and emission measures, which could differ from instrument to instrument even during concurrent observations.

Most of this work was done as part of D. F.'s dissertation, who wishes to thank George Goldenbaum, his dissertation advisor, and the members of the dissertation committee: Mukul Kundu, Adil Hassam, Richard Ellis, Parvez Guzdar, of the University of Maryland, and Joe Davila of GSFC/ NASA. The paper was improved by suggestions from the referee. The research was supported by the NASA suborbital program and NASA grants $879-11-38$ and $170-38$ 52.

\section{REFERENCES}

Arnaud, M., \& Raymond, J. 1992, ApJ, 398, 394

Arnaud, M., \& Rothenflug, R. 1985, A\&AS, 60, 427

Aschwanden, M. J., Newmark, J. S., Delaboudiniere, J.-P., Neupert W. M., Klimchuck J. A., Gary, G. A., Portier-Frozzani, F., \& Zucker, A. 1999, ApJ, 515, 842

Brosius, J. W., et al. 1993, ApJ, 411, 310

Cook, J. W., Chenh, C.-C., Jacobs, V. L., \& Antiochos, S. K. 1989, ApJ, 338, 1176

Falconer, D. A., Davila, J. M., \& Thomas, R. J. 1997, ApJ, 482, 1050

Feldman, U., \& Doschek, G. A. 1977, J. Opt. Soc. Am., 67, 726

Giovanelli, R. 1984, Secrets of the Sun (Cambridge: Cambridge Univ. Press)

Guhathakurta, M., Rottman, G. J., Fisher, R. R., Orral, F. Q., \& Altrock R. C. 1992, ApJ, 388, 633

Lenz, D. D., Deluca E. E., Golub, L., Rosner, R., \& Bookbinder, J. A. 1999, ApJ, 517, L155

Meyer, J. P. 1985, ApJS, 57, 173
Meyer, J.P.1996, in The Sun and Beyond, ed. J.Tran Thanh Van, L. Celnikier, H. C. Trung, \& S. Vauclair, (Gif-sur-Yvette: Editions Frontières), 27

Monsignori-Fossi, B. C., \& Landini, M. 1996, in Astrophysics in the Extreme Ultraviolet, ed. S. Bowyer \& R.F. Malina (Dordrecht: Kluwer), 543

Neupert, W. M., Epstein, G. L., Thomas, R. J., \& Thompson, W.T. 1992, Sol. Phys., 137, 87

Neupert, W.M., et al. 1998, Sol. Phys., 183, 305

Rosner, R., Tucker, W. H., \& Vaiana, G. S. 1978, ApJ, 287, 404

Spitzer, L, Jr. 1962, Physics of Fully Ionized Gases (New York: Interscience)

Thomas, R. J., Keski-Kuha, R. A. M., Neupert, W. M., Condor, C. E., \& Gum, J. S. 1991, Appl. Opt., 30, 2245

Thomas, R. J., \& Neupert W. M. 1994, ApJS, 91, 461

Thompson, W. T., Neupert, W. M., Jordan, S. D., Jones, H. R., Thomas, R. J., \& Schmieder, B. 1993, Sol. Phys., 147, 29

Zirker, J. B. 1993, Sol. Phys., 148, 43 